\title{
Analytical determination of application point and normal reaction arm when rolling a wheel on a drum
}

\author{
Maria Karelina ${ }^{1}$, Tatyana Balabina ${ }^{1}$, and Alexey Mamaev $^{2 *}$ \\ ${ }^{1}$ Moscow Automobile and Road State Technical University (MADI), Moscow, Russia \\ ${ }^{2}$ Moscow Polytechnic University (Moscow Polytech), Moscow, Russia
}

\begin{abstract}
Evaluation of the rolling resistance of car tires is now often performed on drum stands like car tests. This necessitates the study of the mechanics of interaction between the wheel and the drum in order to determine its force and kinematic characteristics, including the values and points of application of tangential and normal forces in contact with the drum. These problems can be solved taking into account that the mechanics of elastic wheel rolling on a drum is the same as when rolling on a flat rigid support surface. In this paper, from consideration of the mechanics of interaction between an elastic wheel and a drum, using the equations of power balance and force equilibrium of the wheel, the equations for determining the point of normal reaction in contact and its arm relative to the wheel axis during its rolling along one and two drums have been derived.. These dependencies have a simple form and can be applied when considering the rolling of both a single wheel and the car as a whole on a drum stand.
\end{abstract}

\section{Introduction}

When testing tires on a drum test stand, as well as cars, one should take into account the change in rolling conditions of the wheels $[1-8]$ compared to rolling on a flat surface. This necessitates the study of the mechanics of interaction between the wheel and the drum in order to determine its force and kinematic characteristics, including the values and points of application of tangential and normal forces in contact with the drum.

These problems can be solved taking into account that the mechanics of elastic wheel rolling on a drum is the same as when rolling on a flat rigid support surface [9-19].

As we know, the normal reaction between the wheel and the supporting surface, on which the wheel rolls, is displaced relative to the axis of the wheel [9-15].

According to V.I. Novopolsky [9], the vertical reaction drift relative to the physical axis and the rolling resistance arm are not identical quantities. Moreover, the drift, as noted by E.A. Chudakov [10] and N.I. Glagolev [11], can have negative values for the braking wheel rolling, which would lead to absurdity.

\footnotetext{
* Corresponding author: am4maev@yandex.ru
} 
V.A. Petrushov [12] proposed to consider the vertical reaction displacement as consisting of two components - the component $b_{H}$ of the normal reaction displacement, resulting from rolling resistance losses due to hysteresis in the tire material, and the component $b_{F}$, resulting from tire deformations due to horizontal force.

In the works of R.V. Virabov [13, 14] expressions are derived to determine the components of the displacement of the normal base reaction. For the driving wheel the normal base reaction is displaced relative to the middle of the contact line by an arm equal to the sum $b_{F}+b_{H}$ (both arms $b_{F}$ and $b_{H}$ have the same sign in this case), for the braking wheel - the difference of these values (the component $b_{F}$ is negative).

Other researchers hold a similar point of view $[15,16]$.

In the case of interaction between a wheel and a rigid drum, as in the case of rolling on a flat support surface, there is a redistribution of normal pressures along the contact area and, as a consequence, a shift of the application point of the normal reaction of the drum relative to the center of the wheel (Fig. 1).

In $[20,21,22]$ equations were derived to calculate the magnitude of the tangential force arising in the contact of the wheel with the drum, the relative loss (slippage) of wheel speed, the power of friction losses in the contact and hysteresis in the wheel material, as well as the rolling resistance factor of the driven wheel. At the same time, the question of the point of application of the normal force in contact with the drum and, accordingly, the arm of this force relative to the wheel axis (Fig.1) was not addressed. These values are necessary, in particular, when considering the rolling of cars during tests on drum stands. Partially, with respect to the tangential reaction, this problem was solved in [22].

Let's consider how we can determine the point of the normal force application in the contact and its arm relative to the wheel axis, considering the joint use of the equations of force equilibrium and power balance of the wheel.

\section{Theory}

According to Fig. 1, the point of application of the normal reaction between the wheel and the drum, defined by the displacement from the middle of the contact area, is found as:

$$
x_{F_{n}}=\left(\arcsin \frac{h_{n}}{r_{p}+r_{d}}\right) r_{d}
$$

where $h_{n}$ is the arm of the normal reaction of the drum relative to the center of the wheel; $r_{\delta}$ - the drum radius, $r_{d}$ - distance from the wheel axis to the drum along the center line $O_{d}$ $O_{K}$.

To determine the arm $h_{n}$ we will use the joint solution of the equations of force equilibrium and power balance of the wheel for different rolling modes - driving, braking and driven one.

\subsection{Driving wheel rolling mode (Fig.1).}

The equations of force equilibrium and power balance of the driving wheel are, respectively, of the form:

$$
\begin{gathered}
M_{\kappa}=F_{t} h_{t}+F_{n} h_{n}, \\
M_{k} w_{k}=P_{f r .}+P_{d} .
\end{gathered}
$$


Here $M_{\kappa}$ is the torque applied to the wheel, $F_{\tau}$ and $h_{\tau}$ re the tangential force in the contact and its arm relative to the wheel axis, $P_{f r}$-the power of friction losses in the contact, $P_{d}-$ the power dissipated from the drum.

Taking into account that power of losses on friction in contact is defined [13, 14, 17 - 22] according to dependence $P_{f r}=F_{t} \xi V=F_{t} \xi \omega_{d} r_{d}$ (here $\xi$ is the slippage (relative wheel speed loss)), and power, carried away from the drum is equal to $P_{d}=M_{d} \omega_{d}$, where $M_{d}=F_{t} r_{d}$ - the torque on the drum, from equation (3) after transformation we get:

$$
F_{t}=\frac{M_{k}}{r} \text { or } M_{k}=F_{t} r
$$

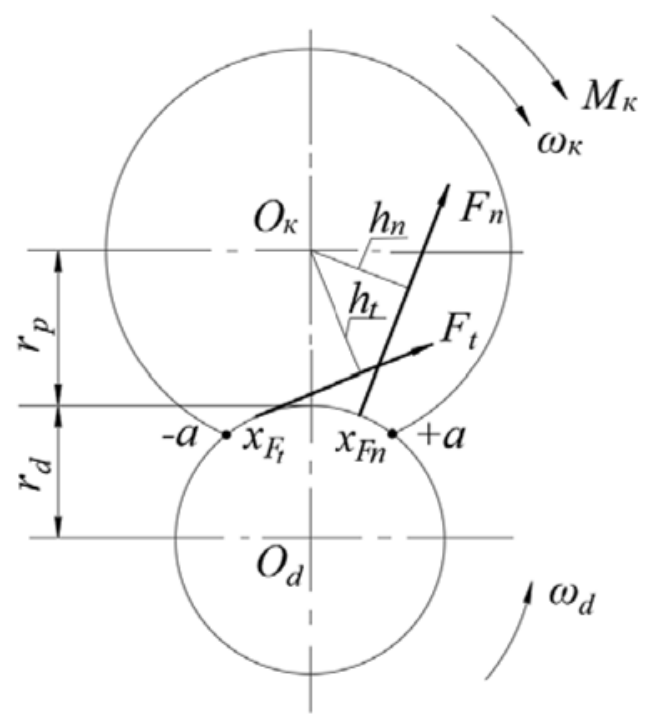

Fig 1. Normal force $F_{n}$ and tangential force $F_{t}$ in the wheel - drum contact and their arms relative to the drum axis.

Substituting the last expression in (2), which connects $M_{k}$ and $F_{t}$, and considering that $F_{t}$ $\approx F_{\tau}$, we get the following:

$$
h_{n}=\frac{F_{t}}{F_{n}}\left(r-h_{\tau}\right)
$$

In the limiting case, when the tangential force in the contact is equal to the limiting force on adhesion, that is $F_{t}=\mu F_{n}$ (where $\mu$ is he coefficient of sliding friction in the contact),

$$
h_{n}^{\text {lim. }}=\mu\left(r-h_{\tau}^{\text {lim. }}\right)=\mu\left(r-r_{d}\right)=\mu W_{0},
$$

where $r$ is the outside wheel radius, $W_{0}$ - wheel deflection along the line $O_{d} O_{K}$ between the axles.

From formula (5) it follows that as the traction force decreases, the arm of the normal base reaction relative to the center of the wheel decreases, since the decrease in $F_{t}$ occurs faster than the growth of the difference $r-h_{\tau}$ due to an increase in the arm $h_{\tau}$. 


\subsection{Braking rolling mode.}

For this case, the equations of force equilibrium and power balance are as follows:

$$
\begin{gathered}
F_{t} h_{t}=M_{T}+h_{n} F_{n}, \\
w_{d} M_{d}=F_{t} r_{d}=P_{f r .}+M_{T} w_{\kappa} .
\end{gathered}
$$

In these equations, the tangential force $F_{\tau}$ and the relative velocity difference $\xi$ (slippage), included in the expression for $P_{f r}$, are taken modulo.

After transformations similar to those in the previous case above, we have:

$$
F_{t}=\frac{M_{T}}{r} \text { or } M_{T}=F_{t} r
$$

Then

$$
h_{n}=\frac{F_{t}}{F_{n}}\left(r-h_{\tau}\right) .
$$

Like for the driving wheel, the limiting arm of the normal reaction of the drum relative to the center of the wheel takes place when the maximum tangential force $F_{t}=\mu F_{n}$ is realized and is equal to the value

$$
h_{n}^{\lim }=\mu W_{0}
$$

Under understanding the positive torque on the wheel as the leading torque and the negative torque as the braking torque, we will present expressions (4) and (9) in general form:

$$
F_{t}=M / r \text { or } M=F_{t} r
$$

Similarly, the expression for the arm of the normal reaction of the drum relative to the center of the wheel can be represented by a general formula if we consider the tangential force acting in the contact of the driving wheel with the drum to be positive, and for the braking wheel - to be negative:

$$
h_{n}=\left(r-h_{\tau}\right) F_{t} / F_{n}
$$

\subsection{Driven rolling mode.}

The rolling resistance of the driven wheel on a flat rigid support surface is determined mainly by hysteresis losses in the tire material.

Similar to rolling a wheel on a flat support surface, the displacement $h_{0}$ of the normal drum reaction due to hysteresis, when rolling on a rigid drum, occurs in the rolling direction of the wheel.

For the driven wheel, the equation of equilibrium is written as:

$$
F_{\tau_{0}} h_{\tau_{0}}=F_{n}\left(h_{0}+h_{n_{0}}\right) \text {, }
$$


where $h_{n_{0}}$ is the arm of the normal reaction of the drum, due to the realization in the contact of the tangential force $F_{\tau_{0}}$, caused by the presence of hysteresis in the wheel material; $h_{\tau_{0}}$ - the arm of the tangential force $F_{\tau_{0}}$. Since the value $F_{\tau_{0}}$ is insignificant, we can approximately accept $x_{F_{\tau_{0}}} \approx-a / 3$ [22], which corresponds to

$$
h_{\tau_{0}}=\left(r_{p}+r_{d}\right) \cos \left(-\frac{a}{3 r_{d}}\right)-r_{d}
$$

Substituting into (14) $h_{n}=h_{n 0}$ according to formula (10), after transformation we have:

$$
F_{\tau_{0}}=F_{n} \frac{h_{0}}{r} .
$$

Using the last expression and formula (10), we find the arm of the normal reaction of the drum, which is due to the realization in the contact of the tangential force $F_{\tau}$, caused by the presence of hysteresis in the wheel material:

$$
h_{n_{0}}=\frac{-F_{\tau_{0}}}{F_{n}}\left(r-h_{\tau_{0}}\right)=\frac{-F_{n}}{F_{n}} \frac{h_{0}}{r}\left(r-h_{\tau_{0}}\right)=h_{0}\left(\frac{h_{\tau_{0}}}{r}-1\right) .
$$

As a result, the total arm of the normal reaction of the drum for the driven rolling mode will be:

$$
h_{0}+h_{n_{0}}=h_{0}+h_{0}\left(\frac{h_{\tau_{0}}}{r}-1\right)=h_{0} \frac{h_{\tau_{0}}}{r} .
$$

Since the product $F_{n} h_{0}$ is the torque $M_{H}$ from hysteresis in the wheel material, by determining the torque $M_{H}$, we can find the value of the arm $h_{0}=M_{H} / F_{n}$.

The torque from the hysteresis in the tire material can be represented as $[20-21]$ :

$$
M_{H}=\frac{P_{H}}{\omega_{K}}=\frac{3}{16} \beta_{H} \alpha F_{n}\left(1+\frac{r}{r_{d}}\right) \text {. }
$$

Then the shift arm of the normal reaction of the drum will be equal to:

$$
h_{0}=\frac{M_{H}}{F_{n}}=\frac{3}{16} \beta_{H} \alpha\left(1+\frac{r}{r_{d}}\right) \text {. }
$$

In works [20 - 22] it is presented that the shift arm of the normal reaction when the wheel rolls on a flat rigid support surface is equal to

$$
b 0=3 \beta_{H} \alpha^{p l} / 16=f_{0} r_{k}^{c} \approx f_{0} r,
$$


where $f_{0}$ is the rolling resistance coefficient of the driven wheel on the plane.

Taking this into account, expression (20) will look like:

$$
h_{0}=f_{0} r\left(1+\frac{r}{r_{p}}\right) \frac{a}{a^{p l}} .
$$

Here $a^{p l}$ is half-length of wheel contact with a flat rigid support surface at the same normal $\operatorname{load} F_{n}$.

According to the last formula, the shoulder of the normal reaction between the wheel and the drum, as well as the rolling resistance of the wheel on the drum, increases by $f_{0}(1+$ $\left.\frac{r}{r_{b}}\right) \frac{\mathrm{a}}{\mathrm{a}^{p l}}$ times compared to the rolling of the wheel on a flat rigid supporting surface.

Let us consider the case when an elastic wheel rolls on two support drums (Fig.2). In particular, this is how the wheels of a car are installed during the running-in tests on drum stands.

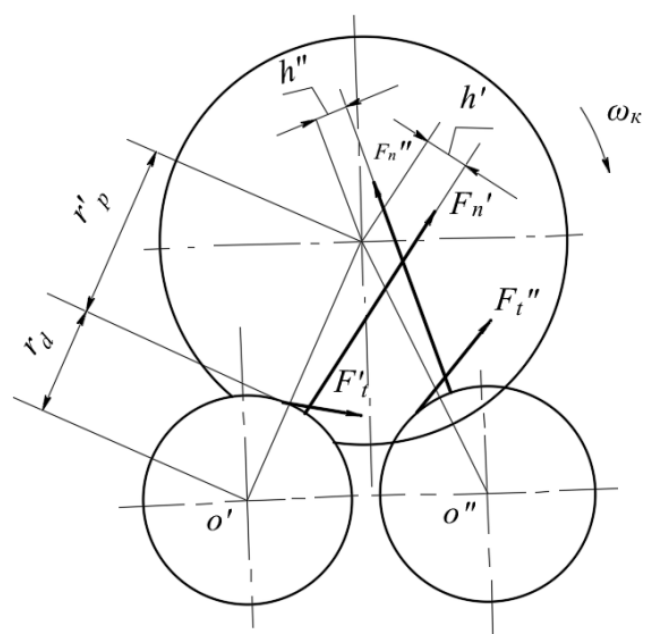

Fig. 2. Normal force $F_{n}$ and tangential force $F_{t}$ in contact of the wheel with the two drums and their arms relative to the axes of the drums.

Let us denote as $\mathrm{e}^{\prime}$ and $\mathrm{e}^{\prime \prime}$ the distance between the center of the wheel and the centers of the left and right drums, respectively:

$$
e^{\prime}=r_{d}^{\prime}+r_{p}^{\prime} \quad e^{\prime \prime}=r_{d}^{\prime \prime}+r_{p}^{\prime \prime}
$$

where $r_{p}^{\prime}$ and $r_{p}^{\prime \prime}$ is the shortest distance from wheel axis to the left and right drums.

Under the action of the torque $M_{\kappa}$ applied to the wheel, the normal reactions of the drums are displaced by the value of $h^{\prime}$ and $h^{\prime \prime}$ :

$$
h^{\prime}=h_{n}^{\prime}+h_{0}^{\prime} h^{\prime \prime}=h_{n}^{\prime \prime}+h^{\prime \prime},
$$

where $h_{n}^{\prime}$ and $h_{n}$ are the shifts of normal reactions due to the implementation of the tangential forces $F_{\tau_{0}}{ }^{\prime}$ and $F_{\tau_{0}}{ }^{\prime \prime}$ in the contact (see formula (13)); $h_{0}^{\prime}$ and $h^{\prime \prime}{ }_{0}$ - normal reaction shifts due to hysteresis in the tire material, which, according to formulas (20) and (21), can be calculated as: 


$$
h_{0}^{\prime(")}=\frac{3}{16} \beta_{p} \alpha^{\prime(")}\left(1+\frac{r}{r_{d}}\right), h_{0}^{\prime\left({ }^{\prime \prime}\right)}=f_{0} r\left(1+\frac{r}{r_{d}}\right) \frac{\alpha^{\prime}\left({ }^{\prime \prime}\right)}{a^{p l\left({ }^{\prime \prime}\right)}}
$$

where $a^{\prime}$ and $a^{\prime \prime}$ are half-length of wheel-drum contact area under the action of normal forces $F_{n}{ }^{\prime}$ and $F_{n}{ }^{\prime \prime} ; a^{p l\left({ }^{\prime \prime}\right)}$ - half-length of the contact area on the plane when the forces $F_{z}^{\prime}$ $=F_{n}{ }^{\prime}$ and $F_{z}^{\prime \prime}=F_{n}{ }^{\prime \prime}$ are acting.

The angles $\gamma_{i}$, which determine the shift of the application point of the normal reactions of the drums, will be found from geometric considerations:

$$
\gamma^{\prime}=\arcsin \frac{h_{n}^{\prime}+h_{0}^{\prime}}{e^{\prime}}, \quad \quad \gamma^{\prime \prime}=\arcsin \frac{h_{n}^{\prime}+h_{0}^{\prime \prime}}{e^{\prime \prime}}
$$

The center of the wheel is asymmetrical with respect to the axes of the drums when the torque $M_{\kappa}$ is applied, here with (Fig.2)

$$
\begin{aligned}
& \alpha^{\prime}=\arccos \frac{\left(e^{\prime}\right)^{2}+c^{2}-\left(e^{\prime \prime}\right)^{2}}{2 e^{\prime} e^{\prime \prime}} \\
& \alpha^{\prime \prime}=\arcsin \left(\frac{e^{\prime} \sin \alpha^{\prime}}{e^{\prime \prime}}\right)
\end{aligned}
$$

\section{Results and conclusions}

Considering the mechanics of interaction between elastic wheel and drum, we have derived equations for determining the point of application of normal reaction in the contact and its arm relative to the wheel axis during its rolling along one and two drums. The derived dependencies show that the arm of the normal reaction between the wheel and the drum, as well as the rolling resistance of the wheel on the drum, increases by $f_{0} r\left(1+\frac{r}{r_{p}}\right) \frac{a}{a^{p l}}$ times compared to rolling the wheel on a flat rigid supporting surface.

These dependencies have a simple form and can be applied when considering the rolling of both a single wheel and the car as a whole on a drum stand.

\section{References}

1. N.Ya. Govoruschenko, Diagnostics of technical condition of cars, Moscow, Transport, p.254 (1970)

2. L.I. Korotkov, Selection of optimal parameters of a test bench for diagnostics of traction and braking properties of cars, Collection, Automobiletransport, Kiev, Technika, 8, p.146 (1971)

3. L.I. Korotkov, Studies of rolling resistance of tires on rolling stands, Automotive industry, 10, pp.13 - 15 (1977)

4. A.V. Serov, Modern car diagnostic stations, NIIavtoprom, p.163 (1971)

5. N.K.Kulikov, Car wheel operation, Works of NAMI, Mashgiz, 77, p.39 (1955)

6. Yu.P.Boroznyak, Determination of rolling resistance of car wheels, Automobile transport, Kiev, Technika, 4, (1968)

7. Yu.P. Boroznyak, Determination of the rolling radius of an automotive wheel in driven mode on a non-deformable surface, Automobiletransport, Kiev, Technika, 3 (1966)

8. B.L Bukhin., Introduction to the mechanics of pneumatic tires, Moscow, Chemistry (1988)

9. V.I. Novopolsky, Experimental study of rolling losses of a car wheel, Automotive and tractor industry, 1 , pp.17 - 20 (1954) 
10. E.A. Chudakov, Automobile theory, Moscow, Mashgiz, p.344 (1950)

11. N.I. Glagolev, Rolling resistance of cylindrical bodies, Applied mathematics and mechanics, 9, pp.318 - 333 (1945)

12. V.A. Petrushov, S.A. Schuklin, V.V. Moskvin, Rolling resistance of cars and trucks, Moscow, Mechanical engineering, p.224 (1975)

13. R.V. Virabov, Rolling of an elastic wheel on a rigid base, Proceedings of Higher Education Institutions, Mechanical engineering, 4, pp.78 - 85 (1967)

14. R.V. Virabov, On estimating the rolling resistance of an elastic wheel on a rigid base, Proceedings of Higher Education Institutions, Mechanical Engineering, 7, pp.45 - 50 (1967)

15. V.I. Knoroz, E.V. Klennikov, I.P. Petrov, A.S. Shelukhin, Yu.M. Yuriev, Car tire operation, Moscow, Transport (1976)

16. V.I. Knoroz, E.V. Klennikov, Tiresandwheels, Moscow, Mechanicalengineering (1975).

17. M.Yu. Karelina, T.A.Balabina, T.Yu. Cherepnina, A.N. Mamaev, A.S. Vorontsov, Influence of Tire Tread Convexity on its Rolling with a Slip, IOP Conference Series: Materials Science and Engineering, 832, p. 012078 (2020)

18. M.Yu. Karelina, T.A. Balabina, T.Yu. Cherepnina, A.N. Mamaev, A. S. Vorontsov, Influence of rolling condition and geometry of tire tread on its wear intensity, IOP Conference Series: Materials Science and Engineering, 832, p. 012079 (2020).

19. T.A. Balabina, Y.I. Brovkina, A.N. Mamaev, Elastic toroidal wheel rolling with side slip, Lecture Notes in Mechanical Engineering, 9783319956299, pp. 2027 - 2035 (2019)

20. A.N. Mamaev, T.A. Balabina, I.V. Odinokova, V.V. Gaevskiy, Interaction mechanics of the wheel with the drum, IOP Conference Series: Materials Science and Engineering, 632 (2019)

21. T.A. Balabina, D.S. Simonov, V.R. Rogov, A.N. Mamaev, A.S. Vorontsov, Determination of Power and Kinematic Wheel Parameters when Rolling against a Drum, IOP Conference Series: Materials Science and Engineering, 832, p. 012077 (2020)

22. M.Yu. Karelina, T.A. Balabina, A.N. Mamaev, Determination of point of application of tangent force in contact of wheel with drum and its arm relative to wheel axis, Proceedings of the 6th International Conference on Industrial Engineering (ICIE 2020), Lecture Notes in Mechanical Engineering (2020) 